Received: 15.01 .2020

Revised: 12.02 .2020

Accepted: 21.02 .2020

DOI: $10.17804 / 2410-9908.2020 .1 .057-072$

\title{
FORMATION OF MAGNETIC PROPERTIES OF ELECTRICAL STEEL
}

\author{
Yu. N. Dragoshanskii ${ }^{\text {a) }}$, V. I. Pudov ${ }^{\text {b)* }}$ \\ M.N. Miheev Institute of Metal Physics, Ural Branch of the Russian Academy of Sciences, \\ 18 S. Kovalevskoy St., Ekaterinburg, 620108, Russian Federation \\ a) iDttps://orcid.org/ 0000-0002-6401-4300 \\ b) iD https://orcid.org/0000-0003-3143-7195 $\otimes$ pudov@imp.uran.ru \\ *Corresponding author. E-mail: pudov@imp.uran.ru \\ Address for correspondence: 18 S. Kovalevskoy St., Ekaterinburg, 620108, Russian Federation \\ Tel.: +7 (343)378 3694
}

The paper studies the influence of the crystal structure on the form and behavior of magnetic domains and on the electromagnetic properties of anisotropic electrical steel based on the $\mathrm{Fe}-3 \% \mathrm{Si}$ alloy with a (110) [001] texture. The physical mechanisms of the rearrangement of the domain type and dynamics during magnetization and stretching of magnetic triple crystallites of various sizes and orientations are considered; the conditions for achieving minimum magnetic losses and an increase in magnetic induction under specified conditions of their magnetization reversal are determined.

Keywords: anisotropic Fe-3 \% Si alloy, crystal structure, domains, magnetic properties.

\section{Acknowledgment}

The work was performed within the state assignment on the subjects of Magnet (No. AAAAA18-118020290129-5) and Diagnostics (No. AAAA-A18-118020690196-3) and supported by a UB RAS Program project.

\section{References}

1. Vonsovskii S.V. Magnetizm [Magnetism]. Moscow, Nauka Publ., 1971, 1032 p.

2. Hubert A., Schafer R. Magnetic Domains, Berlin, Springer, 2009, 686 p.

3. Shilling J.W., House G.L. Magnetic properties and domain structures in grain-oriented 3.2\% Si-Fe. IEEE Trans. Magn., 1974, vol. 10, no 2, pp. 195-223. DOI: 10.1109/TMAG.1974.1058317.

4. Karr B. Magnetostriction. In: Magnetic Properties of Metals and Alloys, Am. Soc. for Metals, Metals Park, Ohio, 1959.

5. Ferro A., Montalenti G., Soardo G.P. Non linearity anomaly of power losses vs frequency in various soft magnetic materials. IEEE Trans. Magn., 1975, vol. 11, no. 5, pp. 1341-1343. DOI: 10.1109/TMAG.1975.1058895.

6. Landau L.D. Sobranie trudov [Collected Papers, ed. by E.M. Lifshits]. Moscow, Nauka Publ., 1969, 128 p. (In Russian).

7. Dragoshanskii Yu.N. Domain structure of three-axis ferromagnets and its contribution to the formation of the properties of soft magnetic alloys. Synopsis of a doctoral dissertation. Ekaterinburg, IFM UrO RAN Publ., 1996, 42 p. (In Russian).

8. Zaikova V.A., Startseva I.E., Filippov B.N. Domennaya struktura i magnitnye svoystva elektrotekhnicheskikh staley [Domain Structure and Magnetic Properties of Electric Steels]. Moscow, Nauka Publ., 1992, 271 p. (In Russian). 
9. Dragoshanskii Yu.N., Pudov V.I. An apparatus for optical monitoring of the surfaces of solids. RF Patent 174673, 2017. (In Russian).

10. Khanzina T.A., Bamburov V.G., Dragoshanskii Yu.N., Vlasova Z.N., Bescherevnikh I.V., Alekseev V.A. A Solution for Applying Insulating Coatings on Steels and the Method of Its Producing. USSR Patent 1608243, 1990. (In Russian).

11. Filippov B.N., Zhakov S.V., Dragoshanskiy Y.N., Starodubtsev Y.N., Lykov E.L. Theory of domain-structures in 3-axial ferromagnetic crystals. The Physics of Metals and Metallography, 1976, vol. 42, no. 2, pp. 260-277.

12. Dragoshanskii Yu.N., Bratuseva E.V., Gubernatorov V.V., Sokolov B.K. Domain width and magnetic loss in grain-oriented soft magnetic materials strained by local bending. The Physics of Metals and Metallography, 1997, vol. 83, no. 3, pp. 270-274.

13. Starodubtsev Y.N, Dragoshanskiy Y.N. Domain size dependence on crystal thickness of silicon-iron. Fizika Metallov i Metallovedenie, vol. 47, iss. 5, pp. 925-931.

14. Dragoshanskiy Y.N., Yesina N.K., Zaykova V.A. Influence of crystallographic texture (110)[001] perfection on magnitude of electromagnetic losses in transformer steel. Fizika Metallov i Metallovedenie, 1978, vol. 45, no. 4, pp. 723-728.

15. Shur Ya. S. and Dragoshanskii Yu. N. On the Shape of Closure Domains inside Fe-Si Crystals. Fiz. Met. Metalloved., 1966, vol. 22 (5), pp. 702-710.

16. Schlenker M. X-ray topographic observation of internal ferromagnetic domain walls. Proceeding of conf. on magnetism (MCM-73), vol. 4. Moscow, Nauka Publ., 1974, pp. 178-186.

17. Degauque J. Len pertes d'4nergie dans les ferromagnetiques metalliques dean: ongines physiques (Energy losses in soft metallic ferromagnetic materials: physical origins). Mem. Erud. Sci. Rev. Metal., 1985, vol. 82, pp. 5-24.

18. Dragoshanskii Y.N., Pudov V.I. Physical Mechanisms of Reverse Magnetization Jumps and Improvement of the Functional Characteristics of Ferromagnetics. Dokl. Phys., 2018, vol. 63, no. 9, pp. 358-361. DOI: 10.1134/S1028335818090045.

19. Dragoshanskii Yu.N. and Shur Ya.S. On the Formation of the Domain Structure of SiliconIron Crystals. Fiz. Met. Metalloved., 1966, vol. 21, no. 5, pp. 678-687.

20. Zhakov S.V., Filippov B.N., and Dragoshanskii Yu.N. Domain Structure and Magnetization Processes in Three-Axial Single Crystals in the Field Applied at an Angle to the Easy Magnetization Direction. Fiz. Met. Metalloved., 1979, vol. 47, no. 2, pp. 310-318.

21. Sasaki T., Imamura M. Magnetostrictive properties of individual grain in grain-oriented 3\% Si-Fe. IEEE Trans. Magn., 1981, vol. 17, no. 6, pp. 2866-2868. DOI: 10.1109/TMAG.1981.1061561.

22. Kirshin A.I., Kalugin A.S., Kratysh G.S. The properties of a cold-rolled Fe-Al alloy band for magnetostrictors. Stal, 1989, no. 10, pp. 70-73. (In Russian).

23. Shur Ya.S., Gubernatorov V.V., Dragoshanskii Yu.N., Baranova N.A., Brishko N.A. A method for increasing magnetostriction in alloys. RF Patent 231565, 1986. (In Russian).

24. Dragoshanskii Yu.N. and Sheiko L.M. Effect of Plane Tensions on the Domain Structure and Magnetic Properties of Silicon Iron. Izv. Akad. Nauk SSSR, Ser. Fiz., 1985, vol. 49, no. 8, pp. 1568-1572. (In Russian).

25. Dragoshanskii Yu.N., Zaikova V.A., Shur Ya.S. Effect of elastic extension on the domain structure of siliceous iron and cobalt crystals. Fizika Metallov i Metallovedenie, 1968, vol. 25, no. 2 , pp. 289-297.

26. Dragoshanskii Yu.N., Zaikova V.A., and Khan E.B. Effect of crystallographic orientation and elastic strain on the electromagnetic loss in Fe-3\%Si single crystals. Trudy mezhdunarodnoy konferentsii po magnetizmu "MKM-73" [Proc. MKM-73 Int. Conf. on Magnetism]. Moscow, Nauka Publ., 1974, vol. 4, pp. 518-522. (In Russian).

27. Dragoshanskii Y.N., Pudov V.I. Optimization by deformation effects of the structure and properties of Fe-Si alloys with different texture. Lett. Mater., 2018, vol. 8 (1), pp. 66-70. DOI: $10.22226 / 2410-3535-2018-1-66-70$. 
28. Nozawa T., Mizogami M., Mogi H., Matsuo Y. Magnetic properties and dynamic domain behavior in grain-oriented 3\% Si-Fe. IEEE Trans. Magn., 1996, vol. 32, no. 2, pp. 572-589. DOI: $10.1109 / 20.486550$.

29. Pudov V.I., Dragoshanskii Y.N. Domain structure and magnetic losses in laminated magnetic circuits upon laser treatment. The Physics of Metals and Metallography, 2015, vol. 116, no. 6, pp. 538-543. DOI: 10.1134/S0031918X15060083.

30. Pudov V.I., Dragoshanskii Y.N. A method of manufacturing anisotropic electrical steel. $R F$ patent 2569260. (In Russian).

31. Pudov V.I., Dragoshanskii Yu.N., Doroshek A.S. Efficiency of local deformation effects on the magnetic structure of core elements. Diagnostics, Resource and Mechanics of materials and structures, 2018, iss. 6, pp. 165-172. DOI: 10.17804/2410-9908.2018.6.165-172. Available at: https://dream-journal.org/issues/2018-6/2018-6_242.html 
Подана в журнал: 15.01.2020

УДК 621.758;537.622

DOI: $10.17804 / 2410-9908.2020 .1 .057-072$

\title{
ФОРМИРОВАНИЕ МАГНИТНЫХ СВОЙСТВ ЭЛЕКТРОТЕХНИЧЕСКОЙ СТАЛИ
}

\author{
Ю. Н. Драгошанский ${ }^{\text {a) }}$, В. И. Пудов ${ }^{\text {)** }}$ \\ Институт физики металлов им. М.Н. Михеева Уральского отделения РАН, \\ С. Ковалевской, 18, 620108, г. Екатеринбург, Российская Федерация

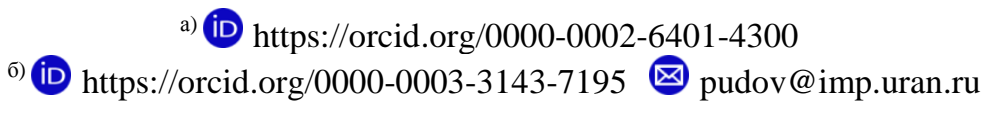 \\ * Ответственный автор. Эл. почта: pudov@imp.uran.ru \\ Адрес для переписки: С. Ковалевской, 18, 620108, Екатеринбург, Российская Федерация \\ Тел.: +7 (343)378-36-94
}

Исследовано влияние кристаллической структуры на вид и поведение магнитных доменов и электромагнитные свойства монокристаллов анизотропной электротехнической стали на основе сплава Fe-3 \% Si с текстурой (110) [001]. Рассмотрены физические механизмы перестройки вида и динамики доменов при намагничивании и растяжении магнитотрехосных кристаллитов различных размеров и ориентаций, а также определены условия достижения минимума магнитных потерь и повышения магнитной индукции при задаваемых условиях их перемагничивания.

Ключевые слова: анизотропный сплав $\mathrm{Fe}-3$ \% $\mathrm{Si}$, кристаллическая структура, домены, магнитные свойства.

\section{1. Введение}

Анизотропная электротехническая сталь (АЭС) состоит из кристаллитов ребровой ориентации (110), рассеяние осей легкого намагничивания [001] которых от продольной оси ленты не превышает нескольких градусов. Отдельные кристаллы имеют размер порядка 10 мм, что позволяет с достаточной достоверностью распространить на АЭС многие закономерности поведения доменных структур, установленные при исследовании монокристаллов.

Основное содержание теоретических, экспериментальных и технологических поисков связано с оптимизацией доменной структуры электротехнической стали. При этом решающее значение принадлежит кристаллографической текстуре и структуре стали, а также особым свойствам ее поверхности, которая при определенных условиях контролирует состояние доменной структуры, а значит, и уровень магнитных свойств.

Цель работы - анализ физических основ для создания современной электротехнической стали с низкими удельными потерями и высокой магнитной индукцией на основе управления доменной структурой, ее видом и условиями перестройки при различных внешних воздействиях. Это в свою очередь связано с анализом результатов изучения кристаллографических и структурных особенностей, а также поверхностных эффектов электротехнической стали.

\section{2. Магнитная энергия и доменная структура}

Ферромагнитные материалы обладают атомным магнитным порядком, при котором магнитные моменты всех носителей магнетизма в веществе параллельны друг другу [1]. При нарушении однородности распределения намагниченности в кристалле плотность энергии обменного взаимодействия возрастает на величину 


$$
\mathrm{E}_{\mathrm{o}}=\mathrm{A}\left[\left(\nabla \alpha_{1}\right)^{2}+\left[\left(\nabla \alpha_{2}\right)^{2}+\left[\left(\nabla \alpha_{3}\right)^{2}\right]\right.\right.
$$

где $\alpha_{i}-$ косинусы вектора спонтанной намагниченности $\overrightarrow{\mathbf{M}}_{\mathrm{s}}: \mathrm{A}$ - постоянная обменного взаимодействия, величина которой в сплаве $\mathrm{Fe}-3 \% \mathrm{Si}$ равна $\sim 1,5 \cdot 10^{-11}$ Дж/м² [2]. Из формулы (1) сле-

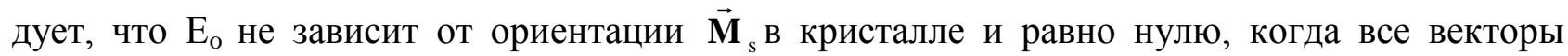
намагниченности параллельны друг другу.

Энергия магнитной анизотропии (ЭМА) проявляется в том, что векторы намагниченности в кристалле ориентируются вдоль определенных направлений, называемых осями легкого намагничивания (ОЛН). В общем случае ЭМА зависит от косинусов $\alpha_{i}$ вектора намагниченности $\overrightarrow{\mathbf{M}}_{\mathrm{s}}$ и компоненты результирующего тензора внешних $\varepsilon_{i \kappa}$ и спонтанных (магнитострикционных) $\varepsilon_{i \kappa}^{0}$ упругих деформаций. В отсутствие внешних деформаций плотность ЭМА $\mathrm{E}_{\mathrm{A}}\left(\alpha_{i}, \varepsilon^{\mathrm{o}}{ }_{i \kappa}\right)$ можно представить в виде

$$
\mathrm{E}_{\mathrm{A}}=\mathrm{E}_{\mathrm{K}}\left(\alpha_{i}\right)+\mathrm{E}_{\mathrm{y}}\left(\varepsilon^{\mathrm{o}}{ }_{i \kappa}\right)+\mathrm{E}_{\mathrm{M}}\left(\alpha_{i}, \varepsilon^{\mathrm{o}}{ }_{i \kappa}\right) .
$$

Здесь $\mathrm{E}_{\mathrm{K}}$ - плотность энергии магнитной кристаллографической анизотропии без учета спонтанных магнитострикционных деформаций, которая устанавливает связь между намагниченностью и кристаллографическими осями. Для кубического кристалла имеем

$$
\mathrm{E}_{\mathrm{K}}=\mathrm{K}_{i}\left(\alpha_{1}^{2} \alpha_{2}^{2}+\alpha_{2}^{2} \alpha_{3}^{2}+\alpha_{1}^{2} \alpha_{3}^{2}\right)+\mathrm{K}_{2} \alpha_{1}^{2} \alpha_{2}^{2} \alpha_{3}^{2},
$$

где $\mathrm{K}_{i}$ и $\mathrm{K}_{2}$ - константы магнитной кристаллографической анизотропии.

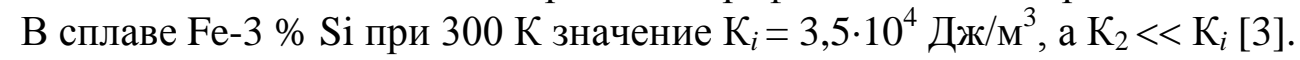

Поэтому минимум $\mathrm{E}_{\mathrm{\kappa}}$ соответствует направлениям $<100>$, которые являются ОЛН кубического кристалла.

Вследствие магнитоупругого взаимодействия кристаллическая решетка ферромагнетика испытывает спонтанную магнитострикционную деформацию. Причем, величина магнитоупругой энергии $\mathrm{E}_{\mathrm{M}}\left(\alpha_{i}, \varepsilon^{\mathrm{o}}{ }_{i \kappa}\right)$ почти линейно снижается с увеличением $\varepsilon^{\mathrm{o}}{ }_{i \kappa}$. Уменьшению же $\mathrm{E}_{\mathrm{M}}$ препятствует рост упругой энергии кристалла $\mathrm{E}_{\mathrm{y}}\left(\varepsilon^{\mathrm{o}}{ }_{i \kappa}\right)$. Если из условия минимума $\mathrm{E}_{\mathrm{A}}$ найти все компоненты магнитострикционных деформаций $\varepsilon_{i \kappa}^{0}$, то относительное удлинение

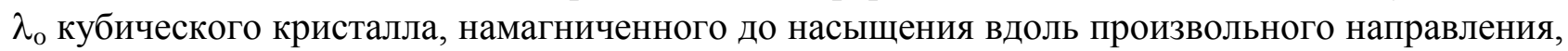
определяемого косинусами $\beta_{i}$, имеет вид

$$
\lambda_{\mathrm{o}}=3 / 2 \lambda_{100}\left(\alpha_{1}^{2} \beta_{1}^{2}+\alpha_{2}^{2} \beta_{2}^{2}+\alpha_{3}^{2} \beta_{3}^{2}-1 / 3\right)-3 \lambda_{111}\left(\alpha_{1} \alpha_{2} \beta_{1} \beta_{2}+\alpha_{2} \alpha_{3} \beta_{2} \beta_{3}+\alpha_{3} \alpha_{1} \beta_{3} \beta_{1}\right) .
$$

Относительное удлинение зависит от величины и знака констант магнитострикции $\lambda_{100}$ и $\lambda_{111}$. Для сплава Fe-3 \% Si при 300 К $\lambda_{100}=23,7 \cdot 10^{-6}$ и $\lambda_{111}=-4,1 \cdot 10^{-6}[1,4]$. При действии однородных внешних напряжений $\sigma_{i \kappa}=\sigma \gamma_{i} \gamma_{\kappa}$ энергия кубического кристалла увеличивается на величину

$$
E_{\sigma}=-3 / 2 \sigma\left[\lambda_{100}\left(\alpha_{1}^{2} \gamma_{1}^{2}+\alpha_{2}^{2} \gamma_{2}^{2}+\alpha_{3}^{2} \gamma_{3}^{2}\right)+2 \lambda_{111}\left(\alpha_{1} \gamma_{1} \alpha_{2} \gamma_{2}+\alpha_{2} \gamma_{3} \alpha_{3} \gamma_{2}+\alpha_{3} \alpha_{1} \gamma_{3} \gamma_{1}\right)\right.
$$

где $\gamma_{1}$ - направляющие косинусы внешнего напряжения б.

Следует отметить, что константы магнитной анизотропии и магнитострикции, а также намагниченность насыщения зависят от содержания кремния и температуры (рис. 1) [1-8]. В частности, сталь с содержанием кремния $\sim 6 \%$ имеет константы магнитострикции $\lambda_{100}, \lambda_{111}$ близки к нулю, что существенно влияет на ее магнитные свойства. 


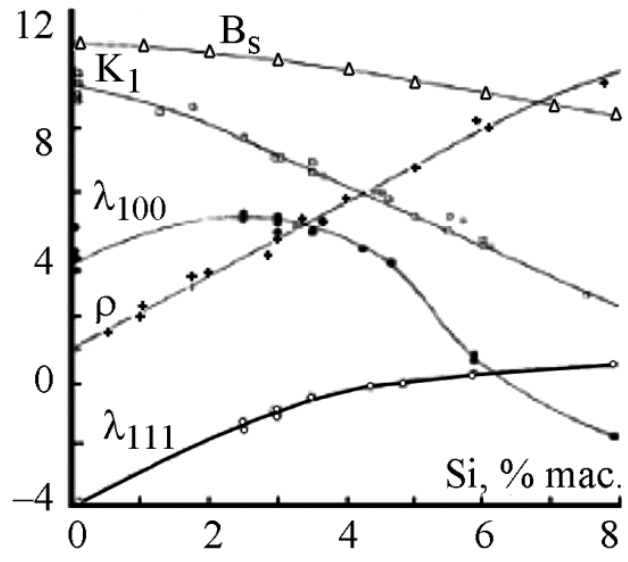

$a$

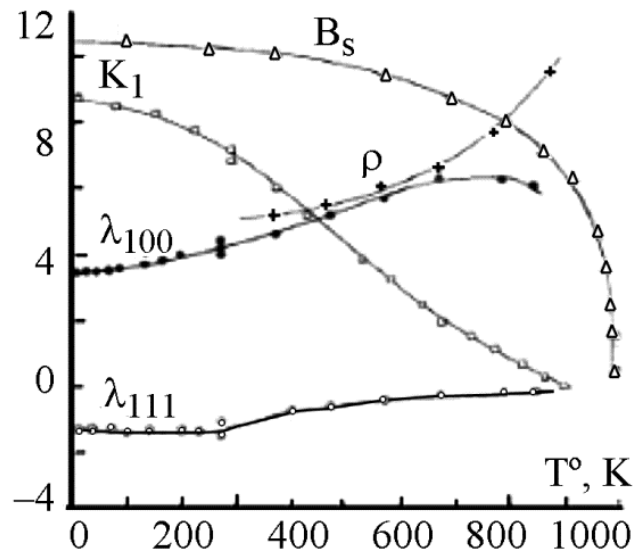

$\sigma$

Рис. 1. Зависимость магнитных констант и электросопротивления от содержания кремния при $\mathrm{T}=300 \mathrm{~K}(a)$ и температуры для $\mathrm{Fe}-3 \% \mathrm{Si}(б)$. Одно деление по оси ординат

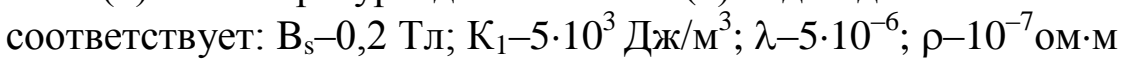

Плотность энергии ферромагнетика во внешнем магнитном поле $\overrightarrow{\mathbf{H}}$

$$
\mathrm{E}_{\mathrm{H}} \approx-\left(\overrightarrow{\mathbf{H}} \overrightarrow{\mathbf{M}}_{\mathrm{s}}\right)
$$

а плотность энергии размагничивающего поля

$$
\mathrm{E}_{\mathrm{H}} \approx-1 / 2\left(\overrightarrow{\mathbf{H}}_{\mathrm{p}} \overrightarrow{\mathbf{M}}_{\mathrm{s}}\right)
$$

где $\overrightarrow{\mathbf{H}}_{\text {р }}$ - магнитное поле поверхностных и объемных магнитных зарядов.

При однородной намагниченности $\operatorname{div} \overrightarrow{\mathbf{M}}=0$ и размагничивающее поле определяется только поверхностными магнитными зарядами с плотностью, равной разности нормальных составляющих намагниченности на границе раздела.

Если ОЛН лежит на плоскости пластины, то на торцевых поверхностях должны возникать магнитные поля рассеяния. Энергия этого поля $\mathrm{E}_{\mathrm{p}}$ в пластинчатом кристалле $\mathrm{Fe}-3 \%$

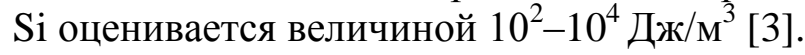

Именно возможность снижения магнитостатической энергии, например, за счет чередования магнитных полюсов различных знаков на торцевой поверхности, приводит к разбиению ферромагнетика на домены - области однородной намагниченности насыщения $\left(\mathrm{M}_{\mathrm{s}}\right)$ [2].

В переходной области между доменами намагниченность постепенно изменяет свое направление. Для кубических кристаллов с тремя ОЛН типичными являются доменные границы (ДГ) Блоха с поворотом намагниченности на $180^{\circ}$ или $90^{\circ}$. В ДГ Блоха вектор намагниченности поворачивается в плоскости ДГ. Они реализуются в кристаллах $\mathrm{Fe}-3 \% \mathrm{Si}$ толщиной более 0,1 мкм. Эффективная ширина и плотность энергии ДГ, отнесенная к единице ее площади, определяется из условия минимума обменной энергии и энергии магнитной анизотропии в переходной области. В кристалле кремнистого железа ДГ имеет эффективную ширину $\sim 10^{-1}$ мкм, плотность энергии $\gamma_{\Gamma} \sim 10^{-3}$ Дж/м ${ }^{3}$, причем $\gamma_{\text {г }}$ для $180^{\circ}$ ДГ с нормалью к направлению [001] в два раза больше, чем для $90^{\circ}$ ДГ [2, 6].

\section{1. Образцы и методы их исследования}

Были исследованы монокристальные пластины АЭС Fe-3 \% Si c ребровой (110) [001] и кубической (100) [001] кристаллографической ориентацией поверхностей. Размеры пла- 
стин: длина 10-120 мм, толщина 0,01-10 мм. Образцы предварительно подвергали электролитическому полированию в растворе хромового ангидрида при $70{ }^{\circ} \mathrm{C}$, плотности тока $2 \mathrm{~A} / \mathrm{cm}^{2}$ площади поверхности образца и отжигали при температуре $1050{ }^{\circ} \mathrm{C}-1$ ч в вакууме. Магнитную доменную структуру выявляли методами порошковых фигур и магнитооптического эффекта Керра. В исследованиях использовали устройство для наблюдения доменов с двух противоположных сторон кристалла одновременно [9].

Напряженность магнитного поля при намагничивании и перемагничивании образцов в различных кристаллографических направлениях в плоскости пластин не превышала $10^{4} \mathrm{~A} / \mathrm{M}$, что обеспечивало перестройку многодоменного состояния размагниченных образцов в однодоменное и обратно.

Одноосное растяжение образцов создавали механически, а плоскостное - нанесением магнитоактивных (растягивающих металл) электроизоляционных покрытий на основе магний-фосфатов с малым коэффициентом теплового расширения $\left(\sim 4-6 \cdot 10^{-6}\right.$ град $\left.^{-1}\right)$, создававших растягивающие напряжения в сплаве до $10 \mathrm{MPa}$ [10].

Линейную магнитострикцию образцов измеряли методом оптического рычага. Удельные магнитные потери лент стали, измеряли в замкнутой магнитной цепи на магнитоизмерительной установке МК-4Э с относительной погрешностью для доверительной вероятности 0,95 не более $\pm 4 \%$.

\section{2. Результаты исследования доменной структуры}

Ориентации поверхностей кристалла относительно ОЛН в значительной степени определяют вид доменной структуры (ДС) в кристалле с тремя ОЛН. Простейшая ДС типа А [8] наблюдается в пластине, на поверхности (100), на которой лежат две ОЛН - [010] и [001] (рис. 2 a).

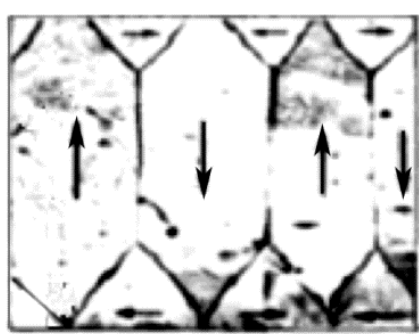

$a$

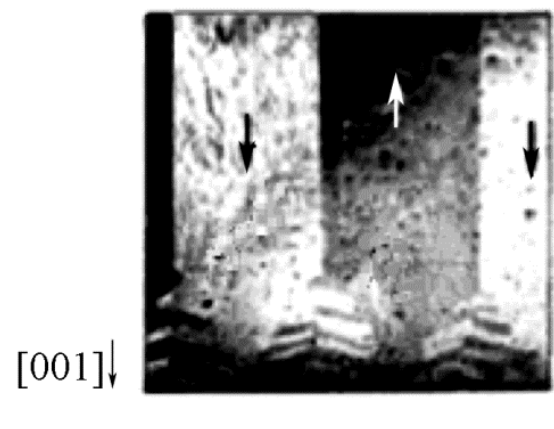

B

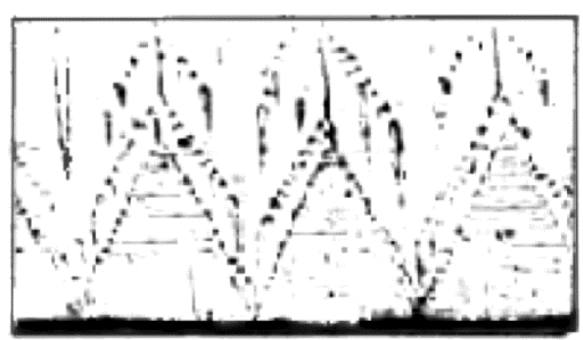

6

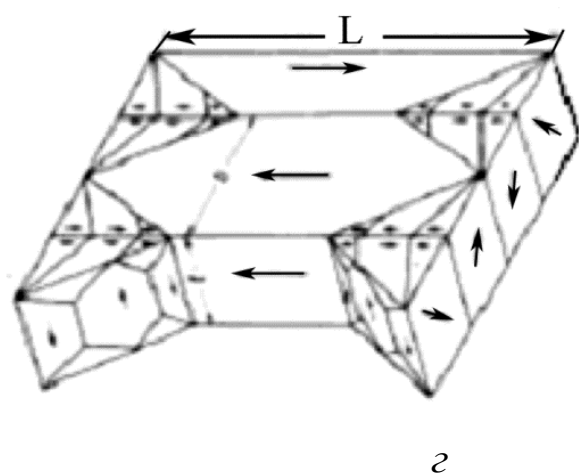

Рис. 2 Вид ДС на поверхности монокристалла (100) (a), (110) $(6$, в) и модель ДГ (2). Толщина кристаллов: $a-0,20 ; \sigma-0,02 ; в-0,15$ мм

В основных доменах, разделенных $180^{\circ}$ ДГ, которые лежат в плоскости (010), намагниченность направлена вдоль оси [001]. Магнитный поток основных доменов вблизи торцевой поверхности замыкается треугольными призматическими доменами. Замыкающие доме- 
ны отделены от основных доменов 90 ДГ по плоскостям (001). Для сплава $\mathrm{Fe}-3$ \% Si характерно, что магнитные поля рассеяния полностью или частично компенсируются замыкающей ДС. Вследствие большой константы магнитной анизотропии этого сплава магнитостатическая энергия значительно больше магнитоупругой энергии и энергии ДГ замыкающей ДС. Поэтому в кристаллах $\mathrm{Fe}-3$ \% Si предпочтительно существование замыкающей ДС. Наличие же трех ОЛН способствует формированию разнообразных видов замыкающей ДС [2, 7].

Равновесное состояние ДС типа А достигается при условии минимума энергии ДГ $\gamma_{\text {г }}$ $\mathrm{L} / \mathrm{D}$ и магнитоупругой энергии $\sim \mathrm{cD}$, связанной с наличием замыкающих треугольных областей

$$
\mathrm{D} \sim \sqrt{ }\left(\gamma_{\mathrm{r}} / \mathrm{c}\right) \mathrm{L},
$$

где L - длина кристалла; D - ширина основных доменов; c - плотность магнитоупругой энергии, которая имеет величину порядка $10^{1}-10^{2}$ Дж/м ${ }^{3}[8]$.

Аналогичная ДС наблюдается на поверхности (110), на которой лежит одна ОЛН-[001], а две другие составляют угол $45^{\circ}$ с поверхностью кристалла (рис. 2 б, в). Основные домены также разделены $180^{\circ}$ ДГ, которые лежат не в плоскости (010), а проходят под углом $232^{\circ}$ к нормали поверхности пластины. С утонением кристалла угол наклона ДГ уменьшается, а при толщинах менее 0,1 мм ДГ проходит перпендикулярно поверхности кристалла [9].

Замыкающие треугольные области представляют скопление доменов с результирующей намагниченностью, направленной навстречу друг другу в соседних комплексах [11]. В относительно тонких кристаллах (мене 0,1 мм) 90 ДГ в замыкающем треугольнике проходят по плоскостям типа [001] (рис. 2 б), а в более толстых - по плоскостям типа [211] (рис. 2 в). Модель ДС (рис. 2 2) хорошо согласуется с доменной структурой, наблюдаемой на трех сопряженных поверхностях кристалла. Зависимость ширины основных доменов также подчиняется соотношению (8), в которое входит толщина кристалла, причем с утонением кристалла ширина основных доменов уменьшается $[11,13]$.

На рис. 3 показано последовательное изменение вида ДС на поверхности кристалла при переходе от плоскости (110) к плоскости (001). Отклонение оси (001) от поверхности (110) на угол $\beta<1^{\circ}$ увеличивает магнитостатическую энергию, которая частично снижается за счет уменьшения ширины основных доменов. При $\beta \approx 1^{\circ}$ по всей поверхности кристалла формируется замыкающая каплевидная ДС (рис. 3 б) [14]. В этом случае магнитостатическая энергия кристалла снижается как за счет образования замыкающей ДС, так и за счет дальнейшего уменьшения ширины основных доменов. На поверхности кристалла замыкающие домены имеют каплевидную форму, намагниченность в которых направлена противоположно намагниченности основного домена [7]. Исследование вида замыкающих доменов на верхней и нижней поверхностях кристалла позволило сделать вывод о связи поверхностных доменов с общим внутренним доменом, намагниченность в котором направлена вдоль [100] или [010] $[9,15]$. Эта модель подтверждена наблюдением ДС методом рентгеновской топографии [16]. По мере приближения «капли» к $180^{\circ}$ ДГ или к соседней «капле» уменьшается длина пути, на которой замыкается магнитный поток, и уменьшается размер каплевидных доменов. Следовательно, размеры замыкающих доменов также подчиняются соотношению (8).

С увеличением угла $\beta$ до $4^{\circ}$ растет плотность замыкающих доменов (рис. 3 в) [17]. «Капли» соединяются друг с другом, образуя упорядоченные ряды в виде «гребенок». Большее увеличение угла $\beta$ приводит к резкому измельчению поверхностной ДС, которая уже слабо отражает характер ДС внутри кристалла (рис. 3 г). На поверхности (111) наблюдается мелкая кружевная ДС (рис. 3 д) [7].

Внутри кристалла сохраняется ДС со $180^{\circ}$ ДГ, расположенными в плоскости (100), а вблизи поверхности магнитный поток замыкается сложной системой замыкающих доменов с $90^{\circ}$ ДГ $[10,13]$. 


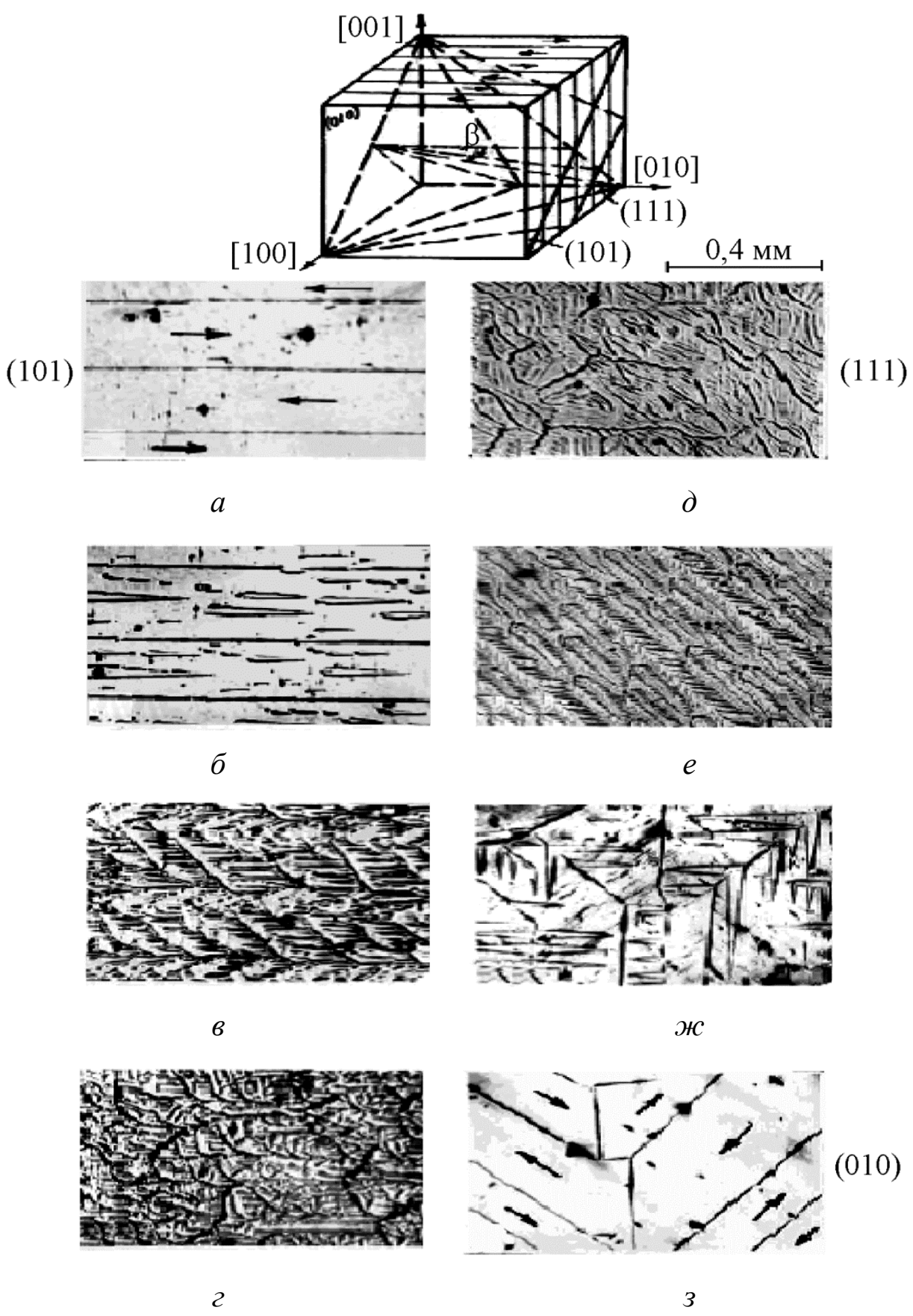

Рис. 3. Изменение вида ДС при переходе от плоскости (101) к плоскости (010) в монокристалле $\mathrm{Fe}-3 \%$ i

Отклонение ОЛН от поверхности (001) приводит к появлению замыкающих доменов в виде «елочек» (рис. 3 ж) [2]. При возрастании наклона ОЛН ветви «елочек» становятся толще и располагаются ближе друг к другу (рис. 3 e), пока не остаются только очертания конечных частей ветвей.

Анизотропия формы кристалла характеризует разницу энергий внешнего магнитного поля, необходимого для намагничивания кристалла вдоль различных ОЛН [8]. Анизотропия формы усиливается с уменьшением размера кристалла вдоль одной из ОЛН, поскольку при этом возрастает энергия магнитных полей рассеяния при намагничивании кристалла до насыщения в этом направлении. Очевидно, что в кристаллической пластине энергетическое преимущество имеет ОЛН, расположенная в плоскости пластины, которую называют 
осью легчайшего намагничивания. По этой причине векторы намагниченности $\overrightarrow{\mathbf{M}}_{\mathrm{s}}$ основных доменов лежат в плоскости пластины и лишь небольшой объем замыкающих доменов имеет векторы намагниченности вдоль двух других ОЛН.

С уменьшением толщины кристалла усиливается анизотропия формы и все больший объем доменов ориентируется вдоль оси легчайшего намагничивания. Поэтому с утонением кристалла вид замыкающей ДС упрощается. На рис. 4 показана ДС в кристалле с $\beta=5^{\circ}$ при различных толщинах [13]. Видно, что наряду с упрощением замыкающей ДС изменяется ширина основных доменов, которая растет вдоль до момента распада рядов «капель» на отдельные домены при $\imath=0,05$ мм. При дальнейшем утонении кристалла резко снижается площадь, занимаемая «каплями», в результате чего проявляется магнитостатическое взаимодействие между основными доменами, которое приводит к снижению D.

В кристалле с ДС типа А, помещенном в магнитное поле в соответствии с формулой (6) минимум энергии имеют домены с $\overrightarrow{\mathbf{M}}_{\mathrm{s}} \uparrow \uparrow \overrightarrow{\mathbf{H}}$, и именно эти домены увеличивают свои размеры. Полевую зависимость магнитной индукции кристалла $\mathrm{B}=\mathrm{B}(\mathrm{H})$ описывает кривая намагничивания. В образце замкнутой формы с направлением магнитного поля вдоль [001] уже при $\mathrm{H}<10$ A/м достигается индукция насыщения $\mathrm{B}_{\mathrm{s}}$ [8]. Величина этого поля соответствует коэрцитивной силе образца $\left(\mathrm{H}_{\mathrm{c}}\right)$, которая определяет магнитное поле задержки смещения $180^{\circ}$ ДГ, вызванной локальными неоднородностями материала [1]. Задержка смещения ДГ приводит к гистерезису перемагничивания кристалла. На петле гистерезиса состояние В = 0 достигается в магнитном поле, также соответствующем $\mathrm{H}_{\mathrm{c}}$.

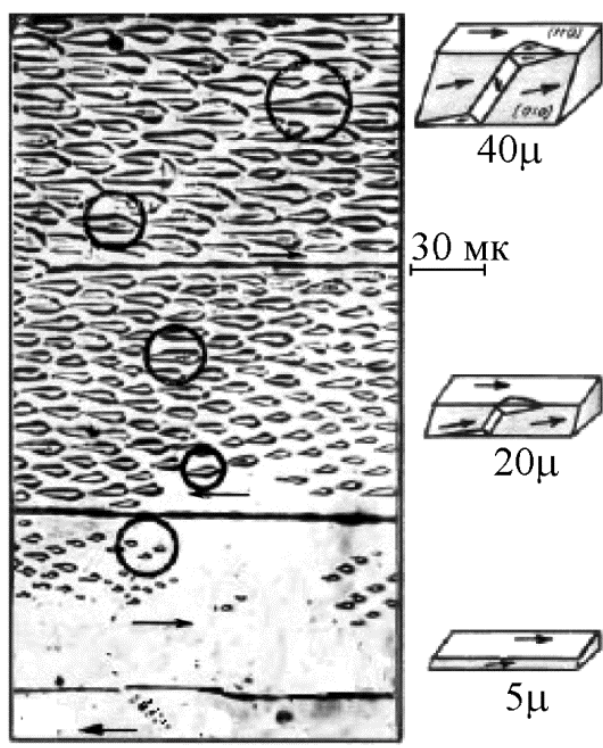

$a$

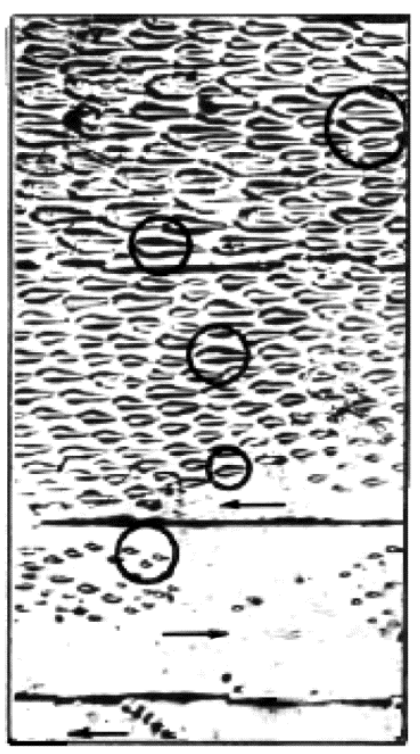

B

Рис. 4. Вид ДС монокристалла на верхней $(a)$ и нижней (б) поверхностях при $\beta=5^{\circ}$ и плавном уменьшении толщины от 40 (в верху) до 5 мкм (внизу)

На схемах представлен объемный вид каплевидных замыкающих комплексов.

В кристалле с поверхностью (110) при направлении магнитного поля вдоль [001] и $\beta>0$ намагничивание в слабых полях также происходит путем смещения $180^{\circ}$ ДГ. Увеличивается площадь поверхности тех основных полосовых доменов, намагниченность которых близка к направлению магнитного поля за счет уменьшения смежных доменов «обратной» намагниченности. При этом в сужающемся основном домене размеры «капель» уменьшаются. В то же время экспансия растущего домена приводит к росту замыкающих каплевидных доменов в нем, а также и к формированию новых каплевидных доменов. Они принимают 
на себя возрастающий магнитный поток на новых территориях растущего основного домена, снижая магнитостатическую энергию кристалла.

Имея направление намагниченности, обратное относительно направления намагниченности растущего основного домена, каждые из новых замыкающих доменов создают при своем возникновении обратные скачки намагниченности (обратные скачки Баркгаузена), природа которых до сих пор оставалась неизвестной. Аналогично, обратные скачки намагниченности создает и исчезновение намагниченных вдоль магнитного поля каплевидных замыкающих доменов в сужающемся основном домене при приближении к ним движущейся $180^{\circ}$ границы. Обратные скачки намагниченности понижают индукцию кристалла в целом. Поэтому повышение степени совершенства кристаллографической текстуры, приводящее к уменьшению объема обратно намагниченных замыкающих областей, обеспечивает рост магнитной индукции сплава, например $\mathrm{B}_{800}$ (индукция в поле $800 \mathrm{~A} / \mathrm{M}$ ), с обычной величины 1,82 Тл в высших марках стали до 1,92-1,96 Тл [18] и более крутой подъем кривой намагничивания (рис. 5).

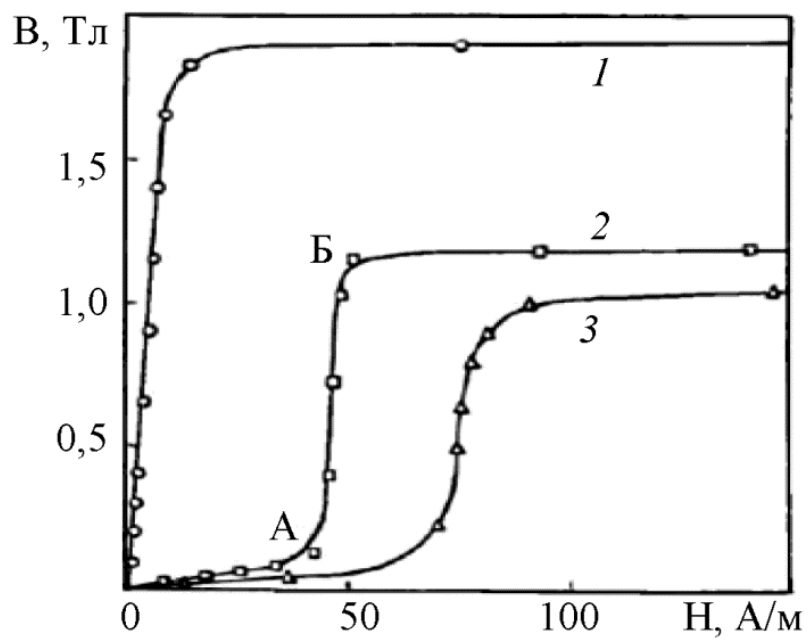

Рис. 5. Кривые намагничивания монокристалла с поверхностью (110) под углом $\alpha$ к направлению [001]: $1-0^{\circ} ; 2-25^{\circ} ; 3-55^{\circ}$

После того как заканчивается смещение $180^{\circ}$ ДГ, что соответствует перегибу на кривой намагничивания, все «капли» имеют практически одинаковый размер. Дальнейшее увеличение магнитного поля приводит к уменьшению объема внутреннего домена в этих замыкающих комплексах до тех пор, пока не останутся только их поверхностные части. В сильном магнитном поле, при котором вектор намагниченности ( $\left.\overrightarrow{\mathbf{M}}_{\mathrm{s}}\right)$ существенно отклоняется от ОЛН к направлению $\overrightarrow{\mathbf{H}}$, исчезает также поверхностная ДС. В кристалле $\mathrm{Fe}-3$ \% Si отклонение $\overrightarrow{\mathbf{M}}_{\mathrm{s}}$ от ОЛН происходит в магнитном поле более $10^{4} \mathrm{~A} / \mathrm{M}$. Такие поля соответствуют области приближения к насыщению.

При уменьшении магнитного поля от состояния насыщения формируются зародыши перемагничивания в участках с наибольшей мгнитостатической энергией, т. е. на границе кристалла (а в поликристаллах еще и на межзеренных границах) [19]. Причем этим зародышем в кристалле с тремя ОЛН является фаза с намагниченностью, перпендикулярной направлению магнитного поля [20]. Вследствие того, что в момент образования доменов с намагниченностью вдоль поперечных ОЛН величина Н еще достаточно велика и появление доменов с $\overrightarrow{\mathbf{M}}_{\mathrm{s}}$ против поля энергетически не оправдано, часть магнитного потока поперечных доменов остается незамкнутой. И только в более слабых полях в этих участках появляются области с $\overrightarrow{\mathbf{M}}_{\mathrm{s}}$ против магнитного поля, которые при дальнейшем снижении величины $\mathrm{H}$ вырастают в домены со $180^{\circ}$ ДГ. Появление ДС типа А приводит к уменьшению объема до- 
менов с намагниченностью вдоль поперечных ОЛН, которые сохраняются только вблизи торцов пластины для замыкания магнитного потока основных доменов.

Вследствие кристаллографической анизотропии намагничивание кристаллов в плоскости (110) под углом $\alpha$ к оси 001 затрудняется (рис. 5) [8].

Смещение $180^{\circ}$ ДГ происходит лишь на начальном участке кривой намагничивания и только при небольших углах $\alpha$ [20]. Поведение ДС при намагничивании кристалла с поверхностью (110) под углом $\alpha=25^{\circ}$ представлено на рис. 6. В размагниченном состоянии магнитный поток основных доменов на границе кристалла замыкается с помощью замыкающих треугольных комплексов КЛМ, МНО, ОПР (рис. 6 a). В магнитном поле замыкающие комплексы с результирующей намагниченностью $\overrightarrow{\mathbf{M}}_{\mathrm{s}} \uparrow \downarrow \overrightarrow{\mathbf{H}}$ постепенно уменьшаются в размере за счет роста соседних комплексов. Этот процесс соответствует участку 0А на кривой намагничивания (рис. 5).

После полного исчезновения замыкающих комплексов с намагниченностью $\overrightarrow{\mathbf{M}}_{\mathrm{s}} \uparrow \downarrow \overrightarrow{\mathbf{H}}$ (рис. 6 б) новая ДС типа С легко распространяется по объему кристалла (рис. 6 b-e). Рост новой ДС происходит путем продвижения фронта КЛМНОПР. При этом замыкающие призмы в соседних треугольных областях 1 и 2, 3 и 4, 5 и 6 соединяются друг с другом. Продвижение фронта новой ДС соответствует участку максимальной проницаемости АБ на кривой намагничивания (рис. 5). Высокая магнитная проницаемость обусловлена тем, что в процессе намагничивания не участвуют замыкающие домены вблизи края кристалла, которые сохраняют магнитостатическую энергию на минимальном уровне. В ДС типа С намагниченность доменов внутри кристалла совпадает с направлениями [100] и [010], а на поверхности наблюдается чередование полос с намагниченностью вдоль [001] и [00і̄] [20].

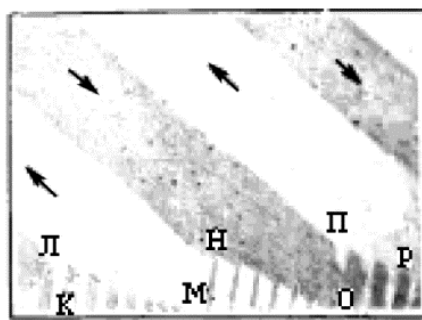

$a$

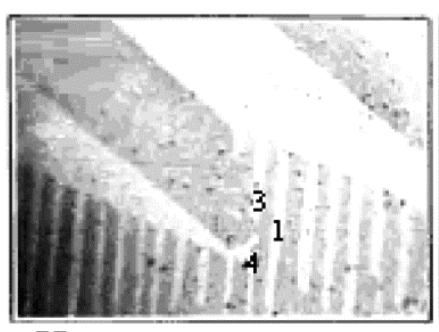

$\mathrm{H}$

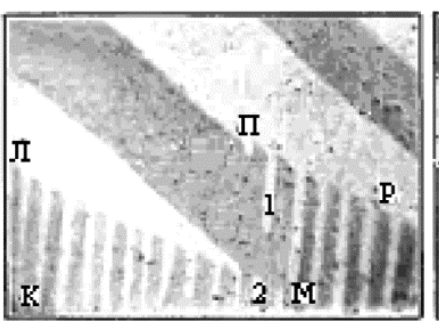

$\sigma$

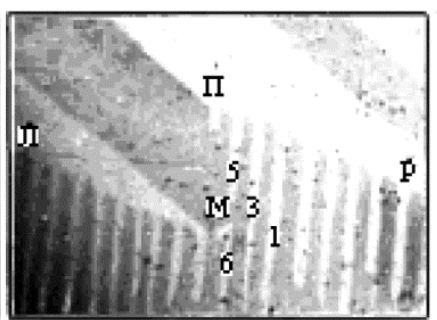

$\partial$

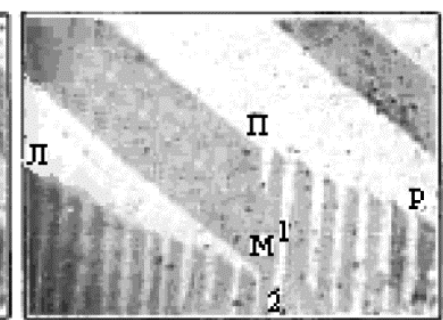

B

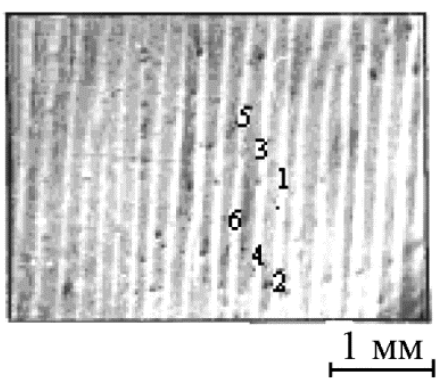

$e$

Рис. 6. Вид ДС на поверхности монокристалла (110) при намагничивании под углом $\alpha=25^{\circ}$. ДС в состояниях $a-\partial$ соответствует точкам кривой намагничивания $\mathrm{O}, \mathrm{A}, \mathrm{Б}$ на рис. 5

При индукции В = 1,0-1,2 Тл, зависящей от толщины кристалла, кристалл полностью заполнен новой ДС типа С и процесс намагничивания снова затрудняется. Увеличение магнитной индукции происходит только за счет уменьшения объема поверхностных замыкающих призм, что приводит к измельчению ДС, и за счет последующего поворота вектора намагниченности $\overrightarrow{\mathbf{M}}_{\text {s }}$ к направлению магнитного поля. 
Такой характер перемагничивания кристалла под углом $\alpha$ к направлению [001] приводит к смещению верхней части петли гистерезиса в сторону больших полей и резкому уменьшению величины остаточной индукции $\mathrm{B}_{\text {ч }}$ [8]. Результирующий вектор намагниченности кристалла с увеличением магнитного поля постепенно отклоняется от направления [001] к направлению $\overrightarrow{\mathbf{H}}$, а при $\alpha>55^{\circ}$ - сначала к направлению [100] ближайшему к полю, а затем снова к $\overrightarrow{\mathbf{H}}$.

В соответствии с формулой (4) перераспределение доменов между тремя ОЛН вызывает деформацию кристалла. Если намагниченность в кристалле ориентирована только вдоль ОЛН, то формулу (4) можно записать в виде $[1,4]$ :

$$
\lambda=3 / 2 \lambda_{100}\left(\sum_{\mathbf{i}=\mathbf{1}}^{\mathbf{3}} \mathbf{n}_{\mathbf{i}} \boldsymbol{\beta}_{\mathbf{i}}^{2}-\frac{\mathbf{1}}{\mathbf{3}}\right)
$$

где $\mathrm{n}_{i}$ - относительный объем кристалла, занимаемый доменами с $\overrightarrow{\mathbf{M}}_{\mathrm{s}}$ вдоль соответствующей ОЛН. Если $\mathrm{n}_{3}=1$, то $\lambda_{1}=\lambda_{100}$. В магнитном поле после образования доменов с $\overrightarrow{\mathbf{M}}_{\mathrm{s}}$ вдоль поперечной оси [100] магнитострикция имеет величину $\lambda_{2}=\lambda_{100}-3 / 2 \lambda_{100} \cdot \mathrm{n}_{1}$, т.е. относительное изменение длины кристалла вдоль оси легчайшего намагничивания [001] составляет $\lambda_{2}-\lambda_{1}=-3 / 2 \lambda_{100} \cdot n_{1}$. Следовательно, образование ДС типа С в магнитном поле соответствует значению продольной магнитострикции, причем $\lambda$ тем меньше, чем больше величина $\mathrm{n}_{1}$.

Если в исходном состоянии без магнитного поля $\mathrm{n}_{3}<1$, т. е. существуют домены с намагниченностью вдоль [100] и [010] (например каплевидные домены), то магнитострикция вдоль направления [001] составляет $\lambda_{1}=3 / 2 \lambda_{100}\left(\mathrm{n}_{3}-1 / 3\right)$. После намагничивания до насыщения $\lambda_{2}=\lambda_{100}$. Тогда магнитострикция насыщения $\lambda_{\mathrm{s}}=3 / 2 \lambda_{100}\left(1-\mathrm{n}_{3}\right)$. Следовательно, $\lambda_{\mathrm{s}}=0$, если намагничивание осуществляется только смещением $180^{\circ}$ ДГ. Положительная величина $\lambda_{\mathrm{s}}$ тем выше, чем больше в исходном состоянии при $\mathrm{H}=0$ объем доменов с намагниченностью вдоль поперечных ОЛН [21]. Проведенные исследования по созданию сжимающих напряжений $\sigma \sim 8 \mathrm{H} / \mathrm{Mм}^{2}$ показало возможность получения подобной доменной структуры в магнитоанизотропных лентах сплавов на основе железа, легированных алюминием или кремнием (например, за счет прокатки ленты, облучения ее поверхности потоком ускоренных ионов, насыщения соответствующими химическими элементами, или нанесением сжимающего покрытия). Это позволило в недорогих, бескобальтовых и безникелевых сплавах на основе железа получить значительную величину продольной магнитострикции насыщения $(60-75) \cdot 10^{-6}$ и изготовить из них мощные эффективные магнитострикционные преобразователи с амплитудой механических колебаний 30-40 мкм [22, 23].

Приложение растягивающих напряжений $\sigma$ изменяет соотношение между магнитоупругой энергией и энергией кристаллографической анизотропии кристалла. При небольших значениях $\sigma<10^{2}$ МПа, когда намагниченность $\overrightarrow{\mathbf{M}}_{\mathrm{s}}$ в доменах ориентирована вдоль ОЛН, напряжение выделяет одну из осей в качестве легчайшей [23].

Одноосное растяжение в плоскости пластины (110), приложенное под углом $0 \leq \alpha<55^{\circ}$ к оси [001], в качестве легчайшей выделяет ось [001]. Если в исходном состоянии кристалл имел ДС типа А, упругое растяжение стабилизирует эту ДС (рис. 7 a) [24]. При этом ширина основных доменов уменьшается (рис. 7 б, в) [25]. Увеличение магнитоупругой энергии замыкающих треугольников под действием $\sigma$ компенсируется уменьшением их размеров, а следовательно, и шириной основных доменов D [25]. Уменьшение ширины доменов приводит к меньшим скоростям движения доменных границ при перемагничивании, вызывая снижение магнитных потерь на вихревые токи [26]. Отметим, что стабильная ориентация $180^{\circ}$ ДГ под влиянием упругого растяжения смещается в сторону нормали к плоскости пластины.

Упругое растяжение вдоль оси [001] уничтожает замыкающие каплевидные доменные комплексы, намагниченность которых неблагоприятно ориентированные относительно $\sigma$, вследствие чего ширина основных доменов снижается еще более значительно (рис. 7 г-e). Исчезновение «капель» начинается с уменьшения объема внутреннего домена, ориентиро- 
ванного вдоль оси [100] или [010]. После этого остаются только поверхностные веретенообразные домены, которые исчезают при дальнейшем увеличении $\sigma[7,25]$.

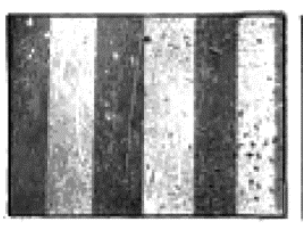

$a$

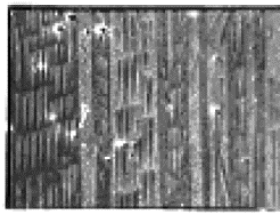

2

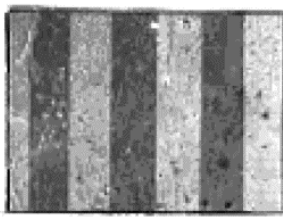

H

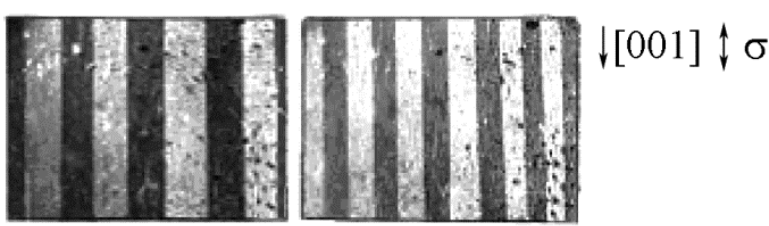

$\sigma$

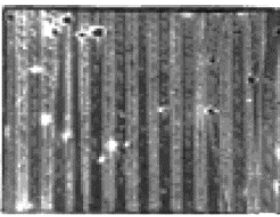

$\partial$

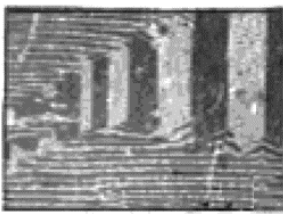

3 $e$

B
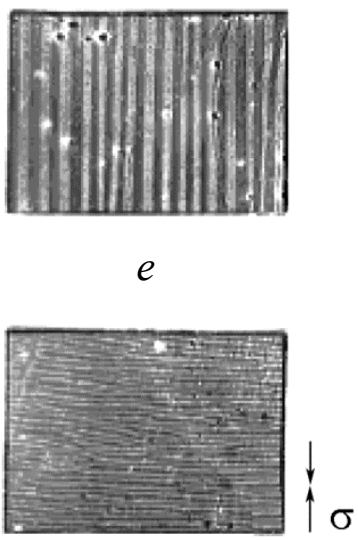

$u$

Рис. 7. Изменение ДС на поверхности монокристалла (110) ( $\beta=0^{\circ}$ для $a-6, \kappa-u$; $\beta=2^{\circ}$ для 2-e) под действием упругого растяжения $(a-e)$ и сжатия (ж-u) вдоль оси [001]. Величина напряжения $\sigma: \mathrm{a}, 2, ж-0 ; \sigma, \partial$, з-5; $, e, u-10 \mathrm{MПа}$

При действии $\sigma$ под углом $55^{\circ}<\alpha \leq 90^{\circ}$ растяжение в качестве оси легчайшего намагничивания выделяет оси [100] или [010] и стабилизирует ДС типа В (рис. $7 u$ ). В ДС типа В внутренние домены намагничены вдоль оси [100] или [010], а магнитный поток замыкается

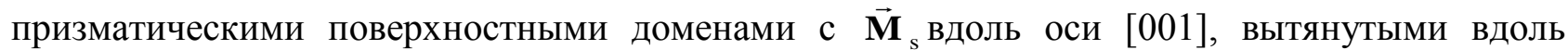
направления [011] [10]. Напряжение, действующее вдоль оси [111] (угол $\alpha \approx 55^{\circ}$ ), не влияет на энергию доменов с намагниченностью $\overrightarrow{\mathbf{M}}_{\text {s }}$ вдоль ОЛН, поэтому ДС и магнитные свойства сплава $\mathrm{Fe}-3 \% \mathrm{Si}$ не изменяются.

Если упругое растяжение и магнитное поле действуют вдоль одной оси при $\alpha<55^{\circ}$, то задерживается формирование ДС типа С. При $\alpha>55^{\circ}$ под действием $\sigma$ образуется ДС типа $\mathrm{B}$, которая существенно облегчает условия перемагничивания кристалла.

Между индукцией и магнитострикцией существует однозначная связь, которая вытекает из дифференциального соотношения для термодинамического потенциала [1].

$$
\left(\frac{\partial \mathbf{B}}{\partial \sigma}\right) \mathrm{H}=4 \pi\left(\frac{\partial \lambda}{\partial \mathrm{H}}\right) \sigma .
$$

Из него следует, что под действием растяжения $\sigma>0$ в области магнитных полей, где $(\partial \lambda \partial H)_{\sigma}<0$, магнитная индукция будет уменьшаться.

При производстве лент АЭС растягивающие напряжения создают нанесением магнитоактивного (растягивающего металла) электроизоляционного покрытия. Плоскостное растяжение покрытия в образцах стали с ребровой текстурой имеет преобладающую компоненту вдоль оси текстуры [7]. Это обеспечивает значительное сужение основных полосовых $180^{\circ}$ доменов и уменьшение магнитных потерь на 5-12 \% в зависимости от степени совершенства текстуры и толщины ленты сплава [27]. 


\section{3. Заключение}

Анализ влияния кристаллоструктурного и текстурного состояния на доменную структуру трехосных ферромагнетиков, проведенный на примере железокремнистых сплавов, открывает пути совершенствования их магнитных свойств. Установлено, что оптимизация вида, размеров, распределения доменов и подвижности их междоменных границ в сплаве $\mathrm{Fe}-3 \% \mathrm{Si}$ приводит к уменьшению удельных магнитных потерь $\mathrm{P}_{1,7 / 50}$ на 10-20 \% и увеличению магнитной индукции $\mathrm{B}_{800}$ на 8-10 \%, по сравнению с характеристиками высших марок АЭС, производимой обычным методом двухстадийной деформации с промежуточным отжигом. Дальнейшие улучшения магнитных свойств электротехнической стали связаны с совершенствованием магнитоактивных покрытий, оптимизацией термомагнитной, лазерной, и других видов обработки [28-31].

\section{Благодарность}

Работа выполнена в рамках ГЗ по темам «Магнит» № АAАA-A18-118020290129-5, «Диагностика» № АААА-А18-118020690196-3 и проекта Программы УрО РАН.

Авторы благодарят Стародубиева Ю.Н. за сбор констант сплава $\mathrm{Fe}$-3 \% $\mathrm{Si}$, изменения которых представлены на рис. 1.

\section{Литература}

1. Вонсовский С. В. Магнетизм. - М. : Наука, 1971. - 1032 с.

2. Hubert A., Schafer R. Magnetic domains. - Berlin : Springer, 2009. - 686 p.

3. Shilling J. W., House G. L. Magnetic properties and domain structures in grain-oriented 3.2\% Si-Fe // IEEE Trans. Magn. - 1974. - Vol. 10, no. 2. - P. 195-223. - DOI: 10.1109/TMAG.1974.1058317.

4. Карр В. Магнитострикция // Магнитные свойства металлов и сплавов / пер. с англ. М. : Изд-во иностр. лит., 1961. - С. 267-327.

5. Ferro A., Montalenti G., Soardo G. P. Non linearity anomaly of power losses vs frequency in various soft magnetic materials // IEEE Trans. Magn. - 1975. - Vol. 11, no. 5. - P. 1341-1343. DOI: 10.1109/TMAG.1975.1058895.

6. Ландау Л. Д. Собрание трудов. Т. 1 / под. ред. Е. М. Лифшица. - М. : Наука, 1969. - 128 с.

7. Драгошанский Ю. Н. Доменная структура трехосных ферромагнетиков и ее роль в формировании свойств магнитомягких сплавов : автореф. дис. ... док. физ.-мат. наук : 01.04.11. - Екатеринбург, 1996. - 142 с.

8. Зайкова В. А., Старцева И. Е., Филиппов Б. Н. Доменная структура и магнитные свойства электротехнических сталей. - М. : Наука, 1992. - 271 с.

9. Устройство для оптического мониторинга поверхности твердых тел : пат. 174673 Рос. Федерация / Драгошанский Ю. Н., Пудов В. И. - № 2017112851/28 ; заявл. 13.04.2017 ; опубл. 25.10. 2017, Бюл. № 30 (II ч.). -5 с.

10. Раствор для нанесения электроизоляционного покрытия на сталь и способ его получения : пат. 1608243 SU / Ханжина Т. А., Бамбуров В. Г., Драгошанский Ю. Н., Власова 3. Н., Бесчеревных И. В., Алексеев В. А. - № 4369481 ; заявл. 28.01.1988 ; опубл. 23.11.1990, Бюл. № 43. - 6 с.

11. Theory of domain-structures in 3-axial ferromagnetic crystals / B. N. Filippov, S. V. Zhakov, Y. N. Dragoshanskiy, Y. N. Starodubtsev, E. L. Lykov // The Physics of Metals and Metallography. - 1976. - Vol. 42, no. 2. - P. 260-277.

12. Domain width and magnetic loss in grain-oriented soft magnetic materials strained by local bending / Yu. N. Dragoshanskii, E. V. Bratuseva, V. V. Gubernatorov, B. K. Sokolov // The Physics of Metals and Metallography. - 1997. - Vol. 83, no. 3. - P. 270-274.

13. Starodubtsev Y. N, Dragoshanskiy Y. N. Domain size dependence on crystal thickness of silicon-iron // Fizika Metallov i Metallovedenie. - Vol. 47, iss. 5. - P. 925-931. 
14. Dragoshanskiy Y. N., Yesina N. K., Zaykova V. A. Influence of crystallographic texture (110)[001] perfection on magnitude of electromagnetic losses in transformer steel // Fizika Metallov i Metallovedenie. - 1978. - Vol. 45, no. 4. - P. 723-728.

15. Shur Ya. S. and Dragoshanskii Yu. N. On the Shape of Closure Domains inside Fe-Si Crystals // Fiz. Met. Metalloved. - 1966. - Vol. 22 (5). - P. 702-710.

16. Schlenker M. X-ray topographic observation of internal ferromagnetic domain walls // Труды международ. конф. по магнетизму (МКМ-73). Т. 4. - М. : Наука, 1974. - С. 178-186.

17. Degauque J. Len pertes d'4nergie dans les ferromagnetiques metalliques dean: ongines physiques (Energy losses in soft metallic ferromagnetic materials: physical origins) // Mem. Erud. Sci. Rev. Metal. - 1985. - Vol. 82. - P. 5-24.

18. Dragoshanskii Y. N., Pudov V. I. Physical Mechanisms of Reverse Magnetization Jumps and Improvement of the Functional Characteristics of Ferromagnetics // Dokl. Phys. - 2018. Vol. 63, no. 9. - P. 358-361. - DOI: 10.1134/S1028335818090045.

19. Dragoshanskii Yu. N. and Shur Ya. S. On the Formation of the Domain Structure of SiliconIron Crystals // Fiz. Met. Metalloved. - 1966. - Vol. 21, no. 5. - P. 678-687.

20. Zhakov S. V., Filippov B. N., and Dragoshanskii Yu. N. Domain Structure and Magnetization Processes in Three-Axial Single Crystals in the Field Applied at an Angle to the Easy Magnetization Direction // Fiz. Met. Metalloved. - 1979. - Vol. 47, no. 2. - P. 310-318.

21. Sasaki T., Imamura M. Magnetostrictive properties of individual grain in grain-oriented 3\% Si-Fe // IEEE Trans. Magn. - 1981. - Vol. 17, no. 6. - P. 2866-2868. - DOI: 10.1109/TMAG.1981.1061561.

22. Киршин А. И., Калугин А. С., Кратыш Г. С. Свойства холоднокатаной ленты из железоалюминиевого сплава для магнитострикторов // Сталь. - 1989. - № 10. - С. 70-73.

23. Способ повышения магнитострикции в сплавах : пат. 231565 SU / Шур Я. С., Губернаторов В. В., Соколов Б. К., Драгошанский Ю. Н., Баранова Н. А., Брышко Н. А. - опубл. 03.02.1986, Бюл. № 43. - 5 с.

24. Драгошанский Ю. Н., Шейко Л. М. Влияние плоскостных растяжений на доменную структуру и магнитные свойства кремнистого железа // Изв. АН СССР. Серия физич. 1985. - T. 49, № 8. - C. 1568-1572.

25. Dragoshanskii Yu. N., Zaikova V. A., Shur Ya. S. Effect of elastic extension on the domain structure of siliceous iron and cobalt crystals // Fizika Metallov i Metallovedenie. - 1968. - Vol. 25, no. 2. - P. 289-297.

26. Драгошанский Ю. Н., Зайкова В. А., Хан Е. Б. Влияние кристаллографической ориентации и упругой деформации на электромагнитные потери монокристаллов $\mathrm{Fe}-3 \% \mathrm{Si} / / \mathrm{T}$ руды Международной конф. МКМ-73. - Т. 4. - М. : Наука, 1974. - С. 518-522.

27. Dragoshanskii Y. N., Pudov V. I. Optimization by deformation effects of the structure and properties of Fe-Si alloys with different texture // Lett. Mater. - 2018. - Vol. 8 (1). - P. 66-70. DOI: 10.22226/2410-3535-2018-1-66-70.

28. Magnetic properties and dynamic domain behavior in grain-oriented 3\% $\mathrm{Si}-\mathrm{Fe} / \mathrm{T}$. Nozawa, M. Mizogami, H. Mogi, Y. Matsuo // IEEE Trans. Magn. - 1996. - Vol. 32, no. 2. - P. 572-589. DOI: $10.1109 / 20.486550$.

29. Pudov V. I., Dragoshanskii Y. N. Domain structure and magnetic losses in laminated magnetic circuits upon laser treatment // The Physics of Metals and Metallography. - 2015. - Vol. 116, no. 6. - P. 538-543. - DOI: 10.1134/S0031918X15060083.

30. Способ изготовления анизотропной электротехнической стали : пат. 2569260 Рос. Федерация / Пудов В. И., Драгошанский Ю. Н. - № 2014113812/02 ; заявл., 8.04.2014; опубл. 20.11.2015, Бюл. № 32. - 4 c.

31. Pudov V. I., Dragoshanskii Yu. N., Doroshek A. S. Efficiency of local deformation effects on the magnetic structure of core elements // Diagnostics, Resource and Mechanics of materials and structures. - 2018. - Iss. 6. - P. 165-172. - DOI: 10.17804/2410-9908.2018.6.165-172. URL: https://dream-journal.org/issues/2018-6/2018-6_242.html 